Korean J. Math. 20 (2012), No. 1, pp. 91-106

\title{
STABILITY OF THE JENSEN FUNCTIONAL EQUATION IN FUZZY BANACH ALGEBRAS
}

\author{
Jung Rye LeE And Dong Yun Shin*
}

\begin{abstract}
In this paper, we prove the Hyers-Ulam stability of the Jensen functional equation in fuzzy Banach algebras by using fixed point method and by using direct method.
\end{abstract}

\section{Introduction and preliminaries}

The theory of fuzzy space has much progressed as developing the theory of randomness. Some mathematicians have defined fuzzy norms on a vector space from various points of view $[1,3,5,6,7,18,19,25,27$, 30, 33, 34, 51]. Following Cheng and Mordeson [11], Bag and Samanta [3] gave an idea of fuzzy norm in such a manner that the corresponding fuzzy metric is of Kramosil and Michalek type [26] and investigated some properties of fuzzy normed spaces [4].

We use the definition of fuzzy normed spaces given in $[3,30,31]$ to investigate a fuzzy version of the Hyers-Ulam stability for the Jensen functional equation in the fuzzy normed algebra setting.

Definition 1.1. $[3,30,31,32]$ Let $X$ be a real vector space. A function $N: X \times \mathbb{R} \rightarrow[0,1]$ is called a fuzzy norm on $X$ if for all $x, y \in X$ and all $s, t \in \mathbb{R}$,

$\left(N_{1}\right) N(x, t)=0$ for $t \leq 0$;

$\left(N_{2}\right) x=0$ if and only if $N(x, t)=1$ for all $t>0$;

$\left(N_{3}\right) N(c x, t)=N\left(x, \frac{t}{|c|}\right)$ if $c \neq 0$

Received January 9, 2012. Revised February 19, 2012. Accepted February 25, 2012 .

2010 Mathematics Subject Classification: 47H10, 46S40, 39B52, 26 E50.

Key words and phrases: fuzzy Banach algebra, fixed point, Hyers-Ulam stability, Jensen functional equation.

This work was supported by the Daejin University Research Grants in 2012.

*Corresponding author. 
$\left(N_{4}\right) N(x+y, s+t) \geq \min \{N(x, s), N(y, t)\} ;$

$\left(N_{5}\right) N(x, \cdot)$ is a non-decreasing function of $\mathbb{R}$ and $\lim _{t \rightarrow \infty} N(x, t)=1$;

$\left(N_{6}\right)$ for $x \neq 0, N(x, \cdot)$ is continuous on $\mathbb{R}$.

The pair $(X, N)$ is called a fuzzy normed vector space.

Definition 1.2. [3, 30, 31, 32] (1) Let $(X, N)$ be a fuzzy normed vector space. A sequence $\left\{x_{n}\right\}$ in $X$ is said to be convergent or converge if there exists an $x \in X$ such that $\lim _{n \rightarrow \infty} N\left(x_{n}-x, t\right)=1$ for all $t>0$. In this case, $x$ is called the limit of the sequence $\left\{x_{n}\right\}$ and we denote it by $N-\lim _{n \rightarrow \infty} x_{n}=x$.

(2) Let $(X, N)$ be a fuzzy normed vector space. A sequence $\left\{x_{n}\right\}$ in $X$ is called Cauchy if for each $\varepsilon>0$ and each $t>0$ there exists an $n_{0} \in \mathbb{N}$ such that for all $n \geq n_{0}$ and all $p>0$, we have $N\left(x_{n+p}-x_{n}, t\right)>1-\varepsilon$.

It is well-known that every convergent sequence in a fuzzy normed vector space is Cauchy. If each Cauchy sequence is convergent, then the fuzzy norm is said to be complete and the fuzzy normed vector space is called a fuzzy Banach space.

We say that a mapping $f: X \rightarrow Y$ between fuzzy normed vector spaces $X$ and $Y$ is continuous at a point $x_{0} \in X$ if for each sequence $\left\{x_{n}\right\}$ converging to $x_{0}$ in $X$, then the sequence $\left\{f\left(x_{n}\right)\right\}$ converges to $f\left(x_{0}\right)$. If $f: X \rightarrow Y$ is continuous at each $x \in X$, then $f: X \rightarrow Y$ is said to be continuous on $X$ (see [4]).

Definition 1.3. Let $X$ be an algebra and $(X, N)$ a fuzzy normed space. if

(1) The fuzzy normed space $(X, N)$ is called a fuzzy normed algebra

$$
N(x y, s t) \geq N(x, s) \cdot N(y, t)
$$

for all $x, y \in X$ and all positive real numbers $s$ and $t$.

(2) A complete fuzzy normed algebra is called a fuzzy Banach algebra.

EXAmple 1.4. Let $(X,\|\cdot\|)$ be a normed algebra. Let

$$
N(x, t)= \begin{cases}\frac{t}{t+\|x\|} & t>0, x \in X \\ 0 & t \leq 0, x \in X .\end{cases}
$$

Then $N(x, t)$ is a fuzzy norm on $X$ and $(X, N(x, t))$ is a fuzzy normed algebra. 
Definition 1.5. Let $\left(X, N_{X}\right)$ and $(Y, N)$ be fuzzy normed algebras. Then a multiplicative $\mathbb{R}$-linear mapping $H:\left(X, N_{X}\right) \rightarrow(Y, N)$ is called a fuzzy algebra homomorphism.

The stability problem of functional equations originated from a question of Ulam [50] concerning the stability of group homomorphisms. Hyers [21] gave a first affirmative partial answer to the question of Ulam for Banach spaces. Hyers' Theorem was generalized by Aoki [2] for additive mappings and by Th.M. Rassias [39] for linear mappings by considering an unbounded Cauchy difference. A generalization of the Th.M. Rassias theorem was obtained by Găvruta [20] by replacing the unbounded Cauchy difference by a general control function in the spirit of Th.M. Rassias' approach.

The functional equation $f(x+y)+f(x-y)=2 f(x)+2 f(y)$ is called a quadratic functional equation. The Hyers-Ulam stability of the quadratic functional equation was proved by Skof [49] for mappings $f$ : $X \rightarrow Y$, where $X$ is a normed space and $Y$ is a Banach space. Cholewa [13] noticed that the theorem of Skof is still true if the relevant domain $X$ is replaced by an Abelian group. Czerwik [14] proved the Hyers-Ulam stability of the quadratic functional equation. The stability problems of several functional equations have been extensively investigated by a number of authors and there are many interesting results concerning this problem (see $[12,15,16,22,24,35]$, [40]-[48]).

TheOrem 1.6. $[8,17]$ Let $(X, d)$ be a complete generalized metric space and let $J: X \rightarrow X$ be a strictly contractive mapping with Lipschitz constant $L<1$. Then for each given element $x \in X$, either

$$
d\left(J^{n} x, J^{n+1} x\right)=\infty
$$

for all nonnegative integers $n$ or there exists a positive integer $n_{0}$ such that

(1) $d\left(J^{n} x, J^{n+1} x\right)<\infty, \quad \forall n \geq n_{0}$;

(2) the sequence $\left\{J^{n} x\right\}$ converges to a fixed point $y^{*}$ of $J$;

(3) $y^{*}$ is the unique fixed point of $J$ in the set $Y=\{y \in X$ $\left.d\left(J^{n_{0}} x, y\right)<\infty\right\}$

(4) $d\left(y, y^{*}\right) \leq \frac{1}{1-L} d(y, J y)$ for all $y \in Y$.

In 1996, G. Isac and Th.M. Rassias [23] were the first to provide applications of stability theory of functional equations for the proof of new fixed point theorems with applications. By using fixed point methods, 
the stability problems of several functional equations have been extensively investigated by a number of authors (see [9, 10, 29], [36]-[38]).

In Section 2, we prove the Hyers-Ulam stability of the Jensen functional equation in fuzzy Banach algebras by using fixed point method. In Section 3, we prove the Hyers-Ulam stability of the Jensen functional equation in fuzzy Banach algebras by using direct method.

Throughout this paper, assume that $\left(X, N_{X}\right)$ is a fuzzy normed algebra and that $(Y, N)$ is a fuzzy Banach algebra.

\section{Hyers-Ulam stability of the Jensen functional equation in fuzzy Banach algebras: fixed point method}

Using fixed point method, we prove the Hyers-Ulam stability of the Jensen functional equation in fuzzy Banach algebras.

Theorem 2.1. Let $\varphi: X^{2} \rightarrow[0, \infty)$ be a function such that there exists an $L<\frac{1}{2}$ with

$$
\varphi(x, y) \leq \frac{L}{2} \varphi(2 x, 2 y)
$$

for all $x, y \in X$. Let $f: X \rightarrow Y$ be a mapping satisfying $f(0)=0$ and

$$
\begin{aligned}
N\left(2 f\left(\frac{r x+r y}{2}\right)-r f(x)-r f(y), t\right) & \geq \frac{t}{t+\varphi(x, y)}, \\
N(f(x y)-f(x) f(y), t) & \geq \frac{t}{t+\varphi(x, y)}
\end{aligned}
$$

for all $x, y \in X$, all $t>0$ and all $r \in \mathbb{R}$. Then $H(x):=N-\lim _{n \rightarrow \infty} 2^{n} f\left(\frac{x}{2^{n}}\right)$ exists for each $x \in X$ and defines a fuzzy algebra homomorphism $H$ : $X \rightarrow Y$ such that

$$
N(f(x)-H(x), t) \geq \frac{(1-L) t}{(1-L) t+\varphi(x, 0)}
$$

for all $x \in X$ and all $t>0$.

Proof. Letting $r=1$ and $y=0$ in (2.1), we get

$$
N\left(2 f\left(\frac{x}{2}\right)-f(x), t\right) \geq \frac{t}{t+\varphi(x, 0)}
$$

for all $x \in X$.

Consider the set

$$
S:=\{g: X \rightarrow Y\}
$$


and introduce the generalized metric on $S$ :

$d(g, h)=\inf \left\{\mu \in \mathbb{R}_{+}: N(g(x)-h(x), \mu t) \geq \frac{t}{t+\varphi(x, 0)}, \forall x \in X, \forall t>0\right\}$,

where, as usual, $\inf \phi=+\infty$. It is easy to show that $(S, d)$ is complete (see the proof of [28, Lemma 2.1]).

Now we consider the linear mapping $J: S \rightarrow S$ such that

$$
J g(x):=2 g\left(\frac{x}{2}\right)
$$

for all $x \in X$.

Let $g, h \in S$ be given such that $d(g, h)=\varepsilon$. Then

$$
N(g(x)-h(x), \varepsilon t) \geq \frac{t}{t+\varphi(x, 0)}
$$

for all $x \in X$ and all $t>0$. Hence

$$
\begin{aligned}
N(J g(x)-J h(x), L \varepsilon t) & =N\left(2 g\left(\frac{x}{2}\right)-2 h\left(\frac{x}{2}\right), L \varepsilon t\right) \\
& =N\left(g\left(\frac{x}{2}\right)-h\left(\frac{x}{2}\right), \frac{L}{2} \varepsilon t\right) \\
& \geq \frac{\frac{L t}{2}}{\frac{L t}{2}+\varphi\left(\frac{x}{2}, 0\right)} \geq \frac{\frac{L t}{2}}{\frac{L t}{2}+\frac{L}{2} \varphi(x, 0)} \\
& =\frac{t}{t+\varphi(x, 0)}
\end{aligned}
$$

for all $x \in X$ and all $t>0$. So $d(g, h)=\varepsilon$ implies that $d(J g, J h) \leq L \varepsilon$. This means that

$$
d(J g, J h) \leq L d(g, h)
$$

for all $g, h \in S$.

It follows from $(2.4)$ that $d(f, J f) \leq 1$.

By Theorem 1.6, there exists a mapping $H: X \rightarrow Y$ satisfying the following:

(1) $H$ is a fixed point of $J$, i.e.,

$$
H\left(\frac{x}{2}\right)=\frac{1}{2} H(x)
$$

for all $x \in X$. The mapping $H$ is a unique fixed point of $J$ in the set

$$
M=\{g \in S: d(f, g)<\infty\} .
$$


This implies that $H$ is a unique mapping satisfying (2.5) such that there exists a $\mu \in(0, \infty)$ satisfying

$$
N(f(x)-H(x), \mu t) \geq \frac{t}{t+\varphi(x, 0)}
$$

for all $x \in X$;

(2) $d\left(J^{n} f, H\right) \rightarrow 0$ as $n \rightarrow \infty$. This implies the equality

$$
N-\lim _{n \rightarrow \infty} 2^{n} f\left(\frac{x}{2^{n}}\right)=H(x)
$$

for all $x \in X$;

(3) $d(f, H) \leq \frac{1}{1-L} d(f, J f)$, which implies the inequality

$$
d(f, H) \leq \frac{1}{1-L}
$$

This implies that the inequality (2.3) holds.

By (2.1),

$$
N\left(2^{k+1} f\left(\frac{r x+r y}{2^{k+1}}\right)-2^{k} r f\left(\frac{x}{2^{k}}\right)-2^{k} r f\left(\frac{y}{2^{k}}\right), 2^{k} t\right) \geq \frac{t}{t+\varphi\left(\frac{x}{2^{k}}, \frac{y}{2^{k}}\right)}
$$

for all $x, y \in X$, all $t>0$ and all $r \in \mathbb{R}$. So

$$
N\left(2^{k+1} f\left(\frac{r x+r y}{2^{k+1}}\right)-2^{k} r f\left(\frac{x}{2^{k}}\right)-2^{k} r f\left(\frac{y}{2^{k}}\right), t\right) \geq \frac{\frac{t}{2^{k}}}{\frac{t}{2^{k}}+\frac{L^{k}}{2^{k}} \varphi(x, y)}
$$

for all $x, y \in X$, all $t>0$ and all $r \in \mathbb{R}$. Since $\lim _{k \rightarrow \infty} \frac{\frac{t}{2^{k}}}{\frac{t}{2^{k}}+\frac{L^{k}}{2^{k}} \varphi(x, y)}=1$ for all $x, y \in X$, all $t>0$ and all $r \in \mathbb{R}$,

$$
N\left(2 H\left(\frac{r x+r y}{2}\right)-r H(x)-r H(y), t\right)=1
$$

for all $x, y \in X$, all $t>0$ and all $r \in \mathbb{R}$. Thus $2 H\left(\frac{r x+r y}{2}\right)-r H(x)-$ $r H(y)=0$. Since $f(0)=0, H(0)=0$. So the mapping $H: X \rightarrow Y$ is additive and $\mathbb{R}$-linear.

By (2.2),

$$
N\left(4^{k} f\left(\frac{x y}{4^{k}}\right)-2^{k} f\left(\frac{x}{2^{k}}\right) \cdot 2^{k} f\left(\frac{y}{2^{k}}\right), 4^{k} t\right) \geq \frac{t}{t+\varphi\left(\frac{x}{2^{k}}, \frac{y}{2^{k}}\right)}
$$


for all $x, y \in X$, all $t>0$ and all $r \in \mathbb{R}$. So

$$
N\left(4^{k} f\left(\frac{x y}{4^{k}}\right)-2^{k} f\left(\frac{x}{2^{k}}\right) \cdot 2^{k} f\left(\frac{y}{2^{k}}\right), t\right) \geq \frac{\frac{t}{4^{k}}}{\frac{t}{4^{k}}+\frac{L^{k}}{2^{k}} \varphi(x, y)}
$$

for all $x, y \in X$ and all $t>0$. Since $\lim _{k \rightarrow \infty} \frac{\frac{t}{4^{k}}}{\frac{t}{4^{k}}+\frac{L^{k}}{2^{k}} \varphi(x, y)}=1$ for all $x, y \in X$ and all $t>0$,

$$
N(H(x y)-H(x) H(y), t)=1
$$

for all $x, y \in X$ and all $t>0$. Thus $H(x y)-H(x) H(y)=0$. So the mapping $H: X \rightarrow Y$ is a fuzzy algebra homomorphism, as desired.

Theorem 2.2. Let $\varphi: X^{2} \rightarrow[0, \infty)$ be a function such that there exists an $L<1$ with

$$
\varphi(x, y) \leq 2 L \varphi\left(\frac{x}{2}, \frac{y}{2}\right)
$$

for all $x, y \in X$. Let $f: X \rightarrow Y$ be a mapping satisfying $f(0)=0,(2.1)$ and (2.2). Then $H(x):=N$ - $\lim _{k \rightarrow \infty} \frac{1}{2^{k}} f\left(2^{k} x\right)$ exists for each $x \in X$ and defines a fuzzy algebra homomorphism $H: X \rightarrow Y$ such that

$$
N(f(x)-H(x), t) \geq \frac{(1-L) t}{(1-L) t+L \varphi(x, 0)}
$$

for all $x \in X$ and all $t>0$.

Proof. Let $(S, d)$ be the generalized metric space defined in the proof of Theorem 2.1.

Consider the linear mapping $J: S \rightarrow S$ such that

$$
J g(x):=\frac{1}{2} g(2 x)
$$

for all $x \in X$.

It follows from (2.4) that

$$
N\left(f(x)-\frac{1}{2} f(2 x), \frac{1}{2} t\right) \geq \frac{t}{t+\varphi(2 x, 0)} \geq \frac{t}{t+2 L \varphi(x, 0)}
$$

for all $x \in X$ and all $t>0$. So $d(f, J f) \leq L$. Hence

$$
d(f, H) \leq \frac{L}{1-L}
$$

which implies that the inequality (2.6) holds.

The rest of the proof is similar to the proof of Theorem 2.1. 


\section{Hyers-Ulam stability of the Jensen functional equation in fuzzy Banach algebras: direct method}

In this section, we prove the Hyers-Ulam stability of the Jensen functional equation in fuzzy Banach algebras by using direct method.

Theorem 3.1. Let $\varphi: X^{2} \rightarrow[0, \infty)$ be a function such that

$$
\sum_{j=0}^{\infty} 4^{j} \varphi\left(\frac{x}{2^{j}}, \frac{y}{2^{j}}\right)<\infty
$$

for all $x, y \in X$. Let $f: X \rightarrow Y$ be a mapping satisfying $f(0)=0$,

$$
\lim _{t \rightarrow \infty} N\left(2 f\left(\frac{r x+r y}{2}\right)-r f(x)-r f(y), t \widetilde{\varphi}(x, y)\right)=1
$$

uniformly on $X^{2}$ for each $r \in \mathbb{R}$, and

$$
\lim _{t \rightarrow \infty} N(f(x y)-f(x) f(y), t \widetilde{\varphi}(x, y))=1
$$

uniformly on $X^{2}$, where

$$
\widetilde{\varphi}(x, y):=\sum_{j=0}^{\infty} 2^{j} \varphi\left(\frac{x}{2^{j}}, \frac{y}{2^{j}}\right)
$$

for all $x, y \in X$. Then $H(x):=N-\lim _{n \rightarrow \infty} 2^{n} f\left(\frac{x}{2^{n}}\right)$ exists for each $x \in X$ and defines a fuzzy algebra homomorphism $H: X \rightarrow Y$ such that if for some $\delta>0, \alpha>0$

$$
N\left(2 f\left(\frac{x+y}{2}\right)-f(x)-f(y), \delta \widetilde{\varphi}(x, y)\right) \geq \alpha
$$

for all $x, y \in X$, then

$$
N(f(x)-H(x), \delta \widetilde{\varphi}(x, 0)) \geq \alpha
$$

for all $x \in X$.

Furthermore, the fuzzy algebra homomorphism $H: X \rightarrow Y$ is a unique mapping such that

$$
\lim _{t \rightarrow \infty} N(f(x)-H(x), t \widetilde{\varphi}(x, 0))=1
$$

uniformly on $X$. 
Proof. Let $r=1$ in (3.2). For a given $\varepsilon>0$, by (3.2), we can find some $t_{0}>0$ such that

$$
N\left(2 f\left(\frac{x+y}{2}\right)-f(x)-f(y), t \varphi(x, y)\right) \geq 1-\varepsilon
$$

for all $t \geq t_{0}$. Letting $y=0$ in (3.7), we get

$$
N\left(2 f\left(\frac{x}{2}\right)-f(x), t \varphi(x, 0)\right) \geq 1-\epsilon
$$

for all $x \in X$.

By induction on $n$, we will show that

$$
N\left(f(x)-2^{n} f\left(\frac{x}{2^{n}}\right), t \sum_{k=0}^{n-1} 2^{k} \varphi\left(\frac{x}{2^{k}}, 0\right)\right) \geq 1-\varepsilon
$$

for all $t \geq t_{0}$, all $x \in X$ and all $n \in \mathbb{N}$.

It follows from (3.8) that (3.9) holds for $n=1$.

Assume that (3.9) holds for $n \in \mathbb{N}$. Then

$$
\begin{gathered}
N\left(f(x)-2^{n+1} f\left(\frac{x}{2^{n+1}}\right), t \sum_{k=0}^{n} 2^{k} \varphi\left(\frac{x}{2^{k}}, 0\right)\right) \\
\geq \min \left\{N\left(f(x)-2^{n} f\left(\frac{x}{2^{n}}\right), t_{0} \sum_{k=0}^{n-1} 2^{k} \varphi\left(\frac{x}{2^{k}}, 0\right)\right),\right. \\
\left.N\left(2^{n} f\left(\frac{x}{2^{n}}\right)-2^{n+1} f\left(\frac{x}{2^{n+1}}\right), 2^{n} t_{0} \varphi\left(\frac{x}{2^{n}}, 0\right)\right)\right\} \\
\geq \min \{1-\varepsilon, 1-\varepsilon\}=1-\varepsilon .
\end{gathered}
$$

This completes the induction argument. Letting $t=t_{0}$ and replacing $n$ and $x$ by $p$ and $\frac{x}{2^{n}}$ in (3.9), respectively, we get

$$
\begin{aligned}
& N\left(2^{n} f\left(\frac{x}{2^{n}}\right)-2^{n+p} f\left(\frac{x}{2^{n+p}}\right), 2^{n} t_{0} \sum_{k=0}^{p-1} 2^{k} \varphi\left(\frac{x}{2^{n+k}}, 0\right)\right) \\
& \geq 1-\varepsilon
\end{aligned}
$$

for all integers $n \geq 0, p>0$.

It follows from (3.1) and the equality

$$
\sum_{k=0}^{p-1} 2^{n+k} \varphi\left(\frac{x}{2^{n+k}}, 0\right)=\sum_{k=n}^{n+p-1} 2^{k} \varphi\left(\frac{x}{2^{k}}, 0\right)
$$


that for a given $\delta>0$ there is an $n_{0} \in \mathbb{N}$ such that

$$
t_{0} \sum_{k=n}^{n+p-1} 2^{k} \varphi\left(\frac{x}{2^{k}}, 0\right)<\delta
$$

for all $n \geq n_{0}$ and $p>0$. Now we deduce from (3.10) that

$$
\begin{aligned}
& N\left(2^{n} f\left(\frac{x}{2^{n}}\right)-2^{n+p} f\left(\frac{x}{2^{n+p}}\right), \delta\right) \\
& \geq N\left(2^{n} f\left(\frac{x}{2^{n}}\right)-2^{n+p} f\left(\frac{x}{2^{n+p}}\right), 2^{n} t_{0} \sum_{k=0}^{p-1} 2^{k} \varphi\left(\frac{x}{2^{n+k}}, 0\right)\right) \\
& \geq 1-\varepsilon
\end{aligned}
$$

for each $n \geq n_{0}$ and all $p>0$. Thus the sequence $\left\{2^{k} f\left(\frac{x}{2^{k}}\right)\right\}$ is Cauchy in $Y$. Since $Y$ is a fuzzy Banach space, the sequence $\left\{2^{n} f\left(\frac{x}{2^{n}}\right)\right\}$ converges to some $H(x) \in Y$. So we can define a mapping $H: X \rightarrow Y$ by $H(x):=N-\lim _{n \rightarrow \infty} 2^{n} f\left(\frac{x}{2^{n}}\right)$, namely, for each $t>0$ and $x \in X$,

$$
\lim _{n \rightarrow \infty} N\left(2^{n} f\left(\frac{x}{2^{n}}\right)-H(x), t\right)=1 .
$$

Fix $t>0$ and $0<\varepsilon<1$. Since $\lim _{n \rightarrow \infty} 2^{n} \varphi\left(\frac{x}{2^{n}}, \frac{y}{2^{n}}\right)=0$, there is an $n_{1}>n_{0}$ such that $t_{0} 2^{n} \varphi\left(\frac{x}{2^{n}}, \frac{y}{2^{n}}\right)<\frac{t}{4}$ for all $n \geq n_{1}$. Hence for each $k \geq n_{1}$, we have

$$
\begin{gathered}
N\left(H\left(\frac{x+y}{2}\right)-H(x)-H(y), t\right) \\
\geq \min \left\{N\left(2 H\left(\frac{x+y}{2}\right)-2^{k+1} f\left(\frac{x+y}{2^{k+1}}\right), \frac{t}{4}\right),\right. \\
N\left(H(x)-2^{k} f\left(\frac{x}{2^{k}}\right), \frac{t}{4}\right), N\left(H(y)-2^{k} f\left(\frac{y}{2^{k}}\right), \frac{t}{4}\right), \\
\left.N\left(2^{k+1} f\left(\frac{x+y}{2^{k+1}}\right)-2^{k} f\left(\frac{x}{2^{k}}\right)-2^{k} f\left(\frac{y}{2^{k}}\right), \frac{t}{4}\right)\right\} .
\end{gathered}
$$

The first three terms on the right-hand side of the above inequality tend to 1 as $k \rightarrow \infty$, and the last term is greater than

$$
N\left(2^{k+1} f\left(\frac{x+y}{2^{k+1}}\right)-2^{k} f\left(\frac{x}{2^{k}}\right)-2^{k} f\left(\frac{y}{2^{k}}\right), t_{0} 2^{k} \varphi\left(\frac{x}{2^{k}}, \frac{y}{2^{k}}\right)\right),
$$


which is greater than or equal to $1-\varepsilon$. Thus

$$
N\left(2 H\left(\frac{x+y}{2}\right)-H(x)-H(y), t\right) \geq 1-\varepsilon
$$

for all $t>0$. Since $N\left(2 H\left(\frac{x+y}{2}\right)-H(x)-H(y), t\right)=1$ for all $t>0$, by $\left(N_{2}\right)$,

$$
2 H\left(\frac{x+y}{2}\right)-H(x)-H(y)=0
$$

for all $x \in X$. Hence the mapping $H: X \rightarrow Y$ is additive.

Let $y=0$ in (3.2). For a given $\varepsilon>0$, by (3.2), we can find some $t_{0}>0$ such that

$$
N\left(2 f\left(\frac{r x}{2}\right)-r f(x), t \varphi(x, 0)\right) \geq 1-\varepsilon
$$

for all $t \geq t_{0}$. It follows from (3.11) that $H(r x)=2 H\left(\frac{r x}{2}\right)=r H(x)$ for all $r \in \mathbb{R}$ and all $x \in X$.

Similarly, it follows from (3.3) that $H(x y)=H(x) H(y)$ for all $x, y \in$ $X$.

Now let for some positive $\delta$ and $\alpha,(3.4)$ holds. Let

$$
\varphi_{n}(x, y):=\sum_{k=0}^{n-1} 2^{k} \varphi\left(\frac{x}{2^{k}}, \frac{y}{2^{k}}\right)
$$

for all $x, y \in X$. Let $x \in X$. By the same reasoning as in the beginning of the proof, one can deduce from (3.4) that

$$
N\left(f(x)-2^{n} f\left(\frac{x}{2^{n}}\right), \delta \sum_{k=0}^{n-1} 2^{n-k} \varphi\left(\frac{x}{2^{k}}, 0\right)\right) \geq \alpha
$$

for all positive integers $n$. Let $t>0$. We have

$$
\begin{aligned}
& N\left(f(x)-H(x), \delta \varphi_{n}(x, 0)+t\right) \\
& \quad \geq \min \left\{N\left(f(x)-2^{n} f\left(\frac{x}{2^{n}}\right), \delta \varphi_{n}(x, 0)\right), N\left(2^{n} f\left(\frac{x}{2^{n}}\right)-H(x), t\right)\right\} .
\end{aligned}
$$

Combining (3.12) and (3.13) and the fact that $\lim _{n \rightarrow \infty} N\left(2^{n} f\left(\frac{x}{2^{n}}\right)-H(x), t\right)=$ 1 , we observe that

$$
N\left(f(x)-H(x), \delta \varphi_{n}(x, 0)+t\right) \geq \alpha
$$


for large enough $n \in \mathbb{N}$. Thanks to the continuity of the function $N(f(x)-H(x), \cdot)$, we see that

$$
N(f(x)-H(x), \delta \widetilde{\varphi}(x, 0)+t) \geq \alpha .
$$

Letting $t \rightarrow 0$, we conclude that

$$
N(f(x)-H(x), \delta \widetilde{\varphi}(x, 0)) \geq \alpha .
$$

To end the proof, it remains to prove the uniqueness assertion. Let $T$ be another additive mapping satisfying (3.5) and (3.6). Fix $c>0$. Given $\varepsilon>0$, by (3.6) for $Q$ and $T$, we can find some $t_{0}>0$ such that

$$
\begin{gathered}
N(f(x)-H(x), t \widetilde{\varphi}(x, 0)) \geq 1-\varepsilon \\
N(f(x)-T(x), t \widetilde{\varphi}(x, 0)) \geq 1-\varepsilon
\end{gathered}
$$

for all $x \in X$ and all $t \geq t_{0}$. Fix some $x \in X$ and find some integer $n_{0}$ such that

$$
t_{0} \sum_{k=n}^{\infty} 2^{k} \varphi\left(\frac{x}{2^{k}}, 0\right)<\frac{c}{2}
$$

for all $n \geq n_{0}$. Since

$$
\begin{aligned}
\sum_{k=n}^{\infty} 2^{k} \varphi\left(\frac{x}{2^{k}}, 0\right) & =2^{n} \sum_{k=n}^{\infty} 2^{(k-n)} \varphi\left(2^{n-k} \frac{x}{2^{n}}, 0\right) \\
& =2^{n} \sum_{l=0}^{\infty} 2^{l} \varphi\left(\frac{1}{2^{l}} \frac{x}{2^{n}}, 0\right)=2^{n} \widetilde{\varphi}\left(2^{-l} \frac{x}{2^{n}}, 0\right)
\end{aligned}
$$


we have

$$
\begin{aligned}
& N(H(x)-T(x), c) \\
& \geq \min \left\{N\left(2^{n} f\left(\frac{x}{2^{n}}\right)-H(x), \frac{c}{2}\right), N\left(T(x)-2^{n} f\left(\frac{x}{2^{n}}\right), \frac{c}{2}\right)\right\} \\
& =\min \left\{N\left(f\left(\frac{x}{2^{n}}\right)-H\left(\frac{x}{2^{n}}\right), \frac{2 c}{2^{n}}\right), N\left(T\left(\frac{x}{2^{n}}\right)-f\left(\frac{x}{2^{n}}\right), \frac{2 c}{2^{n}}\right)\right\} \\
& \geq \min \left\{N\left(f\left(\frac{x}{2^{n}}\right)-H\left(\frac{x}{2^{n}}\right), 2^{-n} t_{0} \sum_{k=n}^{\infty} 2^{k} \varphi\left(\frac{x}{2^{k}}, 0\right)\right),\right. \\
& \left.\qquad N\left(T\left(\frac{x}{2^{n}}\right)-f\left(\frac{x}{2^{n}}\right), 2^{-n} t_{0} \sum_{k=n}^{\infty} 2^{k} \varphi\left(\frac{x}{2^{k}}, 0\right)\right)\right\} \\
& =\min \left\{N\left(f\left(\frac{x}{2^{n}}\right)-H\left(\frac{x}{2^{n}}\right), t_{0} \widetilde{\varphi}\left(\frac{x}{2^{n}}, 0\right)\right),\right. \\
& \left.\quad N\left(T\left(\frac{x}{2^{n}}\right)-f\left(\frac{x}{2^{n}}\right), t_{0} \widetilde{\varphi}\left(\frac{x}{2^{n}}, 0\right)\right)\right\} \\
& \geq 1-\varepsilon .
\end{aligned}
$$

It follows that $N(H(x)-T(x), c)=1$ for all $c>0$. Thus $H(x)=T(x)$ for all $x \in X$.

Similarly, we can obtain the following. We will omit the proof.

TheOrem 3.2. Let $\varphi: X^{2} \rightarrow[0, \infty)$ be a function such that

$$
\widetilde{\varphi}(x, y):=\sum_{n=1}^{\infty} 2^{-n} \varphi\left(2^{n} x, 2^{n} y\right)<\infty
$$

for all $x, y \in X$. Let $f: X \rightarrow Y$ be a mapping satisfying (3.2) and (3.3). Then $H(x):=N$ - $\lim _{n \rightarrow \infty} 2^{-n} f\left(2^{n} x\right)$ exists for each $x \in X$ and defines a fuzzy algebra homomorphism $H: X \rightarrow Y$ such that if for some $\delta>0, \alpha>0$

$$
N\left(2 f\left(\frac{x+y}{2}\right)-f(x)-f(y), \delta \widetilde{\varphi}(x, y)\right) \geq \alpha
$$

for all $x, y \in X$, then

$$
N(f(x)-H(x), \delta \widetilde{\varphi}(x, 0)) \geq \alpha
$$

for all $x \in X$. 


\section{References}

[1] R. P. Agarwal, Y. J. Cho and R. Saadati, On Random Topological Structures, Appl. Anal. Vol. 2011, Article ID 762361, 41 pages doi:10.1155/2011/762361.

[2] T. Aoki, On the stability of the linear transformation in Banach spaces, On the stability of the linear transformation in Banach spaces, J. Math. Soc. Japan 2 (1950), 64-66.

[3] T. Bag and S.K. Samanta, Finite dimensional fuzzy normed linear spaces, J. Fuzzy Math. 11 (2003), 687-705.

[4] T. Bag and S.K. Samanta, Fuzzy bounded linear operators, Fuzzy Sets and Systems 151 (2005), 513-547.

[5] Y. J. Cho, M. Eshaghi Gordji and S. Zolfaghari, Solutions and Stability of Generalized Mixed Type QC Functional Equations in Random Normed Spaces, Inequal. Appl. Vol. 2010, Article ID 403101, pp. 16.

[6] Y. J. Cho, C. Park and R. Saadati, Fuzzy Functional Inequalities, J. Comput. Anal. Appl. 13(2011), 305-320.

[7] Y. J. Cho and R. Saadati, Lattice Non-Archimedean Random Stability of ACQ Functional Equation, Advan. in Diff. Equat. 2011, 2011:31.

[8] L. Cădariu and V. Radu, Fixed points and the stability of Jensen's functional equation, J. Inequal. Pure Appl. Math. 4, no. 1, Art. ID 4 (2003).

[9] L. Cădariu and V. Radu, On the stability of the Cauchy functional equation: a fixed point approach, Grazer Math. Ber. 346 (2004), 43-52.

[10] L. Cădariu and V. Radu, Fixed point methods for the generalized stability of functional equations in a single variable, Fixed Point Theory and Applications 2008, Art. ID 749392 (2008).

[11] S.C. Cheng and J.M. Mordeson, Fuzzy linear operators and fuzzy normed linear spaces, Bull. Calcutta Math. Soc. 86 (1994), 429-436.

[12] Y. Cho, C. Park and R. Saadati, Functional inequalities in non-Archimedean Banach spaces, Appl. Math. Letters 23 (2010), 1238-1242.

[13] P.W. Cholewa, Remarks on the stability of functional equations, Aequationes Math. 27 (1984), 76-86.

[14] S. Czerwik, On the stability of the quadratic mapping in normed spaces, Abh. Math. Sem. Univ. Hamburg 62 (1992), 59-64.

[15] S. Czerwik, The stability of the quadratic functional equation. in: Stability of mappings of Hyers-Ulam type, (ed. Th.M. Rassias and J.Tabor), Hadronic Press, Palm Harbor, Florida, 1994, 81-91.

[16] P. Czerwik, Functional Equations and Inequalities in Several Variables, World Scientific Publishing Company, New Jersey, Hong Kong, Singapore and London, 2002.

[17] J. Diaz and B. Margolis, A fixed point theorem of the alternative for contractions on a generalized complete metric space, Bull. Amer. Math. Soc. 74 (1968), 305309.

[18] S. S. Dragomir, Y. J. Cho and J. K. Kim, Subadditivity of some Functionals associated to Jensen's Inequality with Applications, Taiwan. J. Math. 15(2011), $1815-1828$. 
[19] C. Felbin, Finite dimensional fuzzy normed linear spaces, Fuzzy Sets and Systems 48 (1992), 239-248.

[20] P. Găvruta, A generalization of the Hyers-Ulam-Rassias stability of approximately additive mappings, J. Math. Anal. Appl. 184 (1994), 431-436.

[21] D.H. Hyers, On the stability of the linear functional equation, Proc. Nat. Acad. Sci. U.S.A. 27 (1941), 222-224.

[22] D.H. Hyers, G. Isac and Th.M. Rassias, Stability of Functional Equations in Several Variables, Birkhäuser, Basel, 1998.

[23] G. Isac and Th.M. Rassias, Stability of $\psi$-additive mappings: Appications to nonlinear analysis, Internat. J. Math. Math. Sci. 19 (1996), 219-228.

[24] S. Jung, Hyers-Ulam-Rassias Stability of Functional Equations in Mathematical Analysis, Hadronic Press lnc., Palm Harbor, Florida, 2001.

[25] A.K. Katsaras, Fuzzy topological vector spaces II, Fuzzy Sets and Systems 12 (1984), 143-154.

[26] I. Kramosil and J. Michalek, Fuzzy metric and statistical metric spaces, Kybernetica 11 (1975), 326-334.

[27] S.V. Krishna and K.K.M. Sarma, Separation of fuzzy normed linear spaces, Fuzzy Sets and Systems 63 (1994), 207-217.

[28] D. Mihet and V. Radu, On the stability of the additive Cauchy functional equation in random normed spaces, J. Math. Anal. Appl. 343 (2008), 567-572.

[29] M. Mirzavaziri and M.S. Moslehian, A fixed point approach to stability of a quadratic equation, Bull. Braz. Math. Soc. 37 (2006), 361-376.

[30] A.K. Mirmostafaee, M. Mirzavaziri and M.S. Moslehian, Fuzzy stability of the Jensen functional equation, Fuzzy Sets and Systems 159 (2008), 730-738.

[31] A.K. Mirmostafaee and M.S. Moslehian, Fuzzy versions of Hyers-Ulam-Rassias theorem, Fuzzy Sets and Systems 159 (2008), 720-729.

[32] A.K. Mirmostafaee and M.S. Moslehian, Fuzzy approximately cubic mappings, Inform. Sci. 178 (2008), 3791-3798.

[33] M. Mohammadi, Y. J. Cho, C. Park, P. Vetro and R. Saadati, Random Stability of an Additive-quadratic-quartic Functional Equation, J. Inequal. Appl. Vol. 2010, Article ID 754210, pp. 18 pages.

[34] A. Najati and Y. J. Cho, Generalized Hyers-Ulam Stability of the Pexiderized Cauchy Functional Equation in Non-Archimedean Spaces, Fixed Point Theory Appl. Vol. 2011, Article ID 309026, 11 pages, doi:10.1155/2011/309026.

[35] A. Najati, J. I. Kang and Y. J. Cho, Local Stability of the Pexiderized Cauchy and Jensen's equations in Fuzzy Spaces, J. Inequal. Appl. 2011, 2011:78 doi:10.1186/1029-242X-2011-78.

[36] C. Park, Fixed points and Hyers-Ulam-Rassias stability of Cauchy-Jensen functional equations in Banach algebras, Fixed Point Theory and Applications 2007, Art. ID 50175 (2007).

[37] C. Park, Generalized Hyers-Ulam-Rassias stability of quadratic functional equations: a fixed point approach, Fixed Point Theory and Applications 2008, Art. ID 493751 (2008).

[38] V. Radu, The fixed point alternative and the stability of functional equations, Fixed Point Theory 4 (2003), 91-96. 
[39] Th.M. Rassias, On the stability of the linear mapping in Banach spaces, Proc. Amer. Math. Soc. 72 (1978), 297-300.

[40] Th.M. Rassias, Problem 16; 2, Report of the $27^{\text {th }}$ International Symp. on Functional Equations, Aequationes Math. 39 (1990), 292-293; 309.

[41] Th.M. Rassias, On the stability of the quadratic functional equation and its applications, Studia Univ. Babes-Bolyai XLIII (1998), 89-124.

[42] Th.M. Rassias, The problem of S.M. Ulam for approximately multiplicative mappings, J. Math. Anal. Appl. 246 (2000), 352-378.

[43] Th.M. Rassias, On the stability of functional equations in Banach spaces, J. Math. Anal. Appl. 251 (2000), 264-284.

[44] Th.M. Rassias, On the stability of functional equations and a problem of Ulam, Acta Appl. Math. 62 (2000), 23-130.

[45] Th.M. Rassias and P. Šemrl, On the behaviour of mappings which do not satisfy Hyers-Ulam stability, Proc. Amer. Math. Soc. 114 (1992), 989-993.

[46] Th.M. Rassias and P. Šemrl, On the Hyers-Ulam stability of linear mappings, J. Math. Anal. Appl. 173 (1993), 325-338.

[47] Th.M. Rassias and K. Shibata, Variational problem of some quadratic functionals in complex analysis, J. Math. Anal. Appl. 228 (1998), 234-253.

[48] R. Saadati and C. Park, Non-Archimedean $\mathcal{L}$-fuzzy normed spaces and stability of functional equations, Computers Math. Appl. 60 (2010), 2488-2496.

[49] F. Skof, Proprietà locali e approssimazione di operatori, Rend. Sem. Mat. Fis. Milano 53 (1983), 113-129.

[50] S. M. Ulam, A Collection of the Mathematical Problems, Interscience Publ. New York, 1960.

[51] J.Z. Xiao and X.H. Zhu, Fuzzy normed spaces of operators and its completeness, Fuzzy Sets and Systems 133 (2003), 389-399.

Department of Mathematics

Daejin University

Kyeonggi 487-711, Korea

E-mail: jrlee@daejin.ac.kr

Department of mathematics

University of Seoul

Seoul 130-743, Korea

E-mail: dyshin@uos.ac.kr 